\title{
Asporin Expression on Stromal Cells and/or Cancer Cells Might Be A Useful Prognostic Marker in Patients with Diffuse-Type Gastric Cancer
}

\author{
Masakazu Yashiro ${ }^{a-c}$ Tsuyoshi Hasegawa ${ }^{a, b}$ Yurie Yamamoto ${ }^{a, c}$
}

Gen Tsujio ${ }^{a, b}$ Sadaaki Nishimura ${ }^{a, b}$ Tomohiro Sera $^{a, b}$ Atsushi Sugimoto $^{a, b}$

Shuhei Kushiyamaa, ${ }^{a}$ Hiroaki Kasashimaa,b Tatsunari Fukuoka ${ }^{a-c}$

Katsunobu Sakurai $^{\mathrm{b}}$ Takahiro Toyokawab Naoshi Kubo ${ }^{\mathrm{b}}$ Masaichi Ohirab

${ }^{a}$ Molecular Oncology and Therapeutics, Osaka City University Graduate School of Medicine, Osaka City, Japan;

${ }^{b}$ Department of Gastroenterological Surgery, Osaka City University Graduate School of Medicine, Osaka City, Japan;

'Cancer Center for Translational Research, Osaka City University Graduate School of Medicine, Osaka City, Japan

\section{Keywords}

Asporin · Stromal cells · Gastric cancer

\begin{abstract}
Background: Asporin (ASPN), a member of the proteoglycan family, has been shown to have a close correlation with cancer progression. It is not known whether ASPN is an oncogenic driver or a tumor suppressor in human gastric cancer. We sought herein to determine the relationship between ASPN expression and clinicopathological features of gastric cancer. Patients and Methods: A total of 296 gastric cancer patients (diffuse type, $n=144$; intestinal type, $n=152$ ) were enrolled. The ASPN expression level in each case was analyzed by immunohistochemistry. Results: ASPN was mainly found on stromal cells, especially on fibroblasts in tumor stroma, i.e., cancer-associated fibroblasts. The ASPN expression on either cancer cells or stromal cells was significantly
\end{abstract}

high in macroscopic scirrhous-type tumors $(p<0.001)$ and histologically abundant stroma-type tumors ( $p<0.001)$. Interestingly, a Kaplan-Meier survival curve of the 144 cases of diffuse-type gastric cancer revealed a significantly poorer prognosis in patients with ASPN-positive expression ( $p=$ 0.043; log rank) compared to those with ASPN-negative expression, but the prognoses were not significantly different in these subgroups of the 152 cases of intestinal-type gastric cancer. A multivariate analysis with respect to overall survival showed that ASPN expression on stromal cells and/or cancer cells was significantly correlated with overall survival in patients with diffuse-type gastric cancer ( $p=0.041)$. Conclusion: In gastric cancer, ASPN was expressed mainly on stromal cells and partially on cancer cells. ASPN expression on stromal cells and/or cancer cells might be a useful prognostic marker in patients with diffuse-type gastric cancer.

(c) 2021 S. Karger AG, Basel karger@karger.com www.karger.com/esr (c) 2021 S. Karger AG, Base 


\section{Introduction}

Asporin (ASPN) has been recognized as a member of the family of class I small leucine-rich repeat proteoglycans and a major noncollagen component of the extracellular matrix [1-3]. It was recently reported that there is a close correlation between ASPN and cancer progression [4]. ASPN might play a negative role or a positive role in the pathogenesis of different cancers, because ASPN expression has been reported to be positively or negatively correlated with cancer proliferation and migration by regulating different signaling pathways in each cancer type [4]. For example, ASPN acts as an oncogene in some types of cancer, including gastric cancer $[5,6]$, pancreatic cancer [7, 8], colorectal cancer [9], and prostate cancer [10], whereas ASPN acts as a tumor suppressor gene in triple-negative breast cancer [11-13].

It was also reported that ASPN is produced in cancer cells or stromal cells, and most of the relevant studies showed that ASPN is produced from stromal cells in the tumor microenvironment [4]. We demonstrated that ASPN secreted from cancer-associated fibroblasts (CAF) activates the invasion activity of scirrhous gastric cancer cells, one of the specific types of diffuse-type gastric cancer [5], via paracrine effects. However, it is not known whether ASPN is an oncogenic driver or a tumor suppressor in human gastric cancer (including diffuse-type cancer and intestinal-type cancer). We conducted the present study to determine the relationship between ASPN expression and clinicopathological features of gastric cancer, including diffuse-type cancer and intestinal-type cancer.

\section{Materials and Methods}

\section{Patients}

We examined surgical samples from patients at the Osaka City University Hospital, Osaka, Japan. A total of 296 patients who had undergone resection of primary gastric tumors and were confirmed histologically to have gastric cancer were enrolled in this study. None of the patients had undergone preoperative radiation or chemotherapy. The median follow-up time for all 296 patients was 48.2 months (range 2-90). The median follow-up time for the patients who died of the disease was 19.9 months, compared to 60.5 months for surviving patients. The follow-up schedule after gastrectomy was managed according to the Japanese guideline [14], as follows: computed tomography (CT) was performed at intervals of 6-12 months, measurement of tumor markers of CEA and CA19-9 was performed at intervals of 3-6 months, and endoscopy was performed at intervals of 2 years.

Pathological diagnoses and classifications were made according to the Japanese classification of gastric carcinoma (14th edi- tion) [15]. Depth of tumor invasion, histological type, lymph node metastasis, venous invasion, and lymphatic invasion were based on microscopic examination of materials obtained by surgical resection. Histological subtypes were classified as intestinal and a diffuse; the intestinal type includes papillary adenocarcinoma, well-differentiated tubular adenocarcinoma, and moderately-differentiated adenocarcinoma, and the diffuse type includes poorly differentiated adenocarcinoma, signet ring cell carcinoma, and mucinous carcinoma.

\section{Immunohistochemical Techniques}

Paraffine-embedded sections were deparaffinized in xylene and dehydrated through graded ethanol. The sections were heated for $4 \mathrm{~min}$ at $125^{\circ} \mathrm{C}$ by autoclave in Target Retrieval Solution (DAKO, Carpinteria, CA, USA). Then the sections were incubated with $3 \%$ hydrogen peroxide to block endogenous peroxidase activity. The sections were then incubated with anti-ASPN antibody (HPA008435, 1: 750; Sigma Life science, St. Louis, MO, USA) for $30 \mathrm{~min}$ at room temperature. The sections were incubated with biotinylated goat anti-rabbit immunoglobulin $\mathrm{G}$ for $30 \mathrm{~min}$, followed by 3 washes with PBS. The slides were treated with streptavidin-peroxidase reagent and incubated in PBS diaminobenzidine and $1 \%$ hydrogen peroxide (volume/volume), followed by counterstaining with Mayer hematoxylin. The Anti-ASPN antibody (HPA008435) used in this study was validated in 2 independent studies for immunohistochemistry $[5,16]$.

\section{Immunohistochemical Determination}

ASPN expression was evaluated on cancer cells and stromal cells individually based on the intensity of staining and the extent of staining at the invading tumor front. An intensity value of 0 denoted undetectable staining, whereas values of 1 and 2 denoted a low and high immunoreactivity, respectively. Regarding the extent of staining, values of 1 or 2 denoted samples in which $<50 \%$ or $50 \% \geq$ of the tissue showed immunoreactivity correspondingly. The results obtained using these 2 scales were then added together, yielding a single value from 0 to 4 , i.e., the score. In both cancer cells and stromal cells, the cut-off point was determined to be 2; ASPN expression was considered positive when scores were $\geq 3$ and negative when scores were $\leq 2$. The evaluation was made by 2 independent observers without knowledge of the clinical data and histologic diagnoses. When a discrepant evaluation between the 2 independent observers was found, the evaluation was rechecked and discussed and the final score was obtained by consensus.

\section{Statistical Analysis}

The statistical significance of the associations between ASPN expression and various clinicopathological variables was evaluated using a $\chi^{2}$ test or the Fisher exact test. The survival durations were calculated using the Kaplan-Meier method and analyzed by the log-rank test to compare the cumulative survival durations in the patient groups. In addition, the Cox proportional hazards model was used to compute univariate and multivariate HR for the study parameters. In all of the tests, $p<0.05$ was considered statistically significant. SPSS software (SPSS Japan, version 22; Tokyo, Japan) was used for the analysis. 


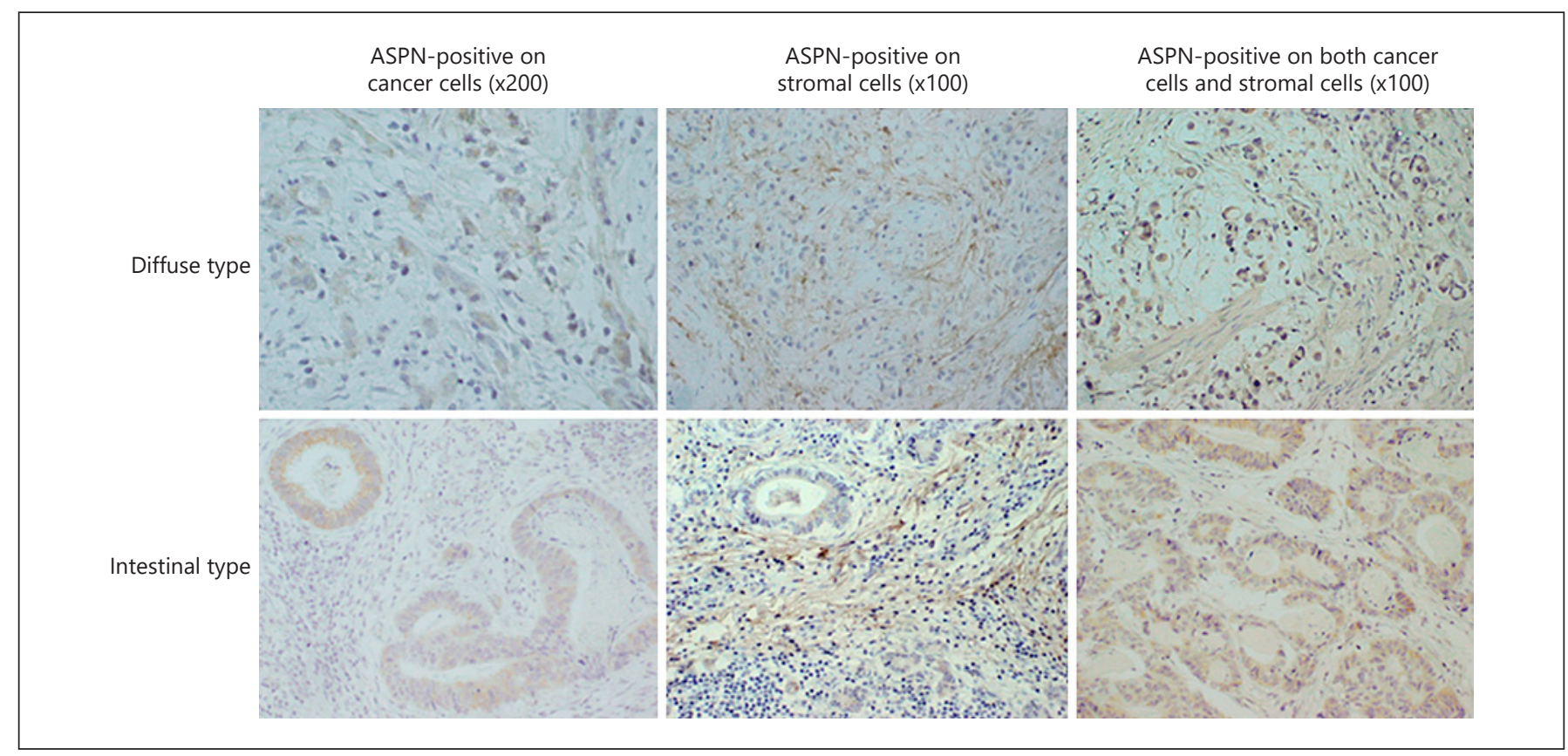

Fig. 1. ASPN expression in diffuse-type cancer and intestinal-type cancer. ASPN was expressed at the cytosol of cells in the gastric tumor environment. In both diffuse-type cancer (top) and intestinal-type cancer (bottom), ASPN was expressed on cancer cells and/or stromal cells, especially on fibroblasts.
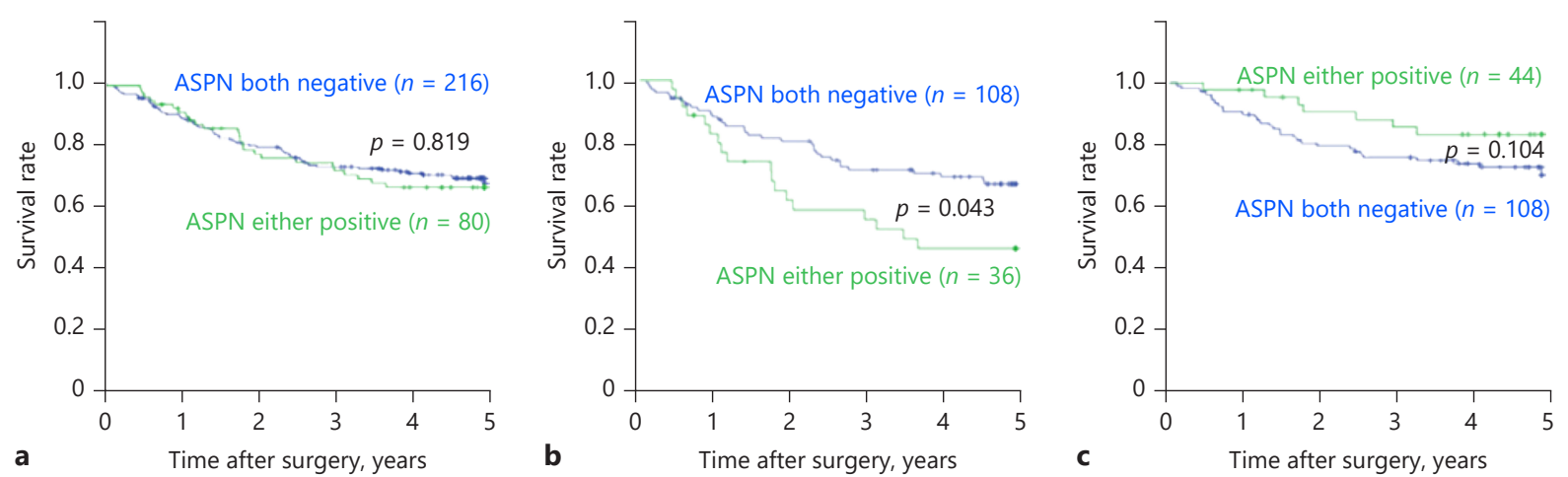

Fig. 2. Survival of patients with gastric cancer. a Kaplan-Meier survival curve for all 296 patients; no correlation was observed between the ASPN-positive and ASPN-positive groups ( $p=0.819)$. b Among the 144 patients with diffuse-type cancer, the prognosis of patients with ASPN-positive expression was significantly poorer than that of patients with ASPN-negative expression $(p=0.043)$. c Among the 152 cases of intestinal-type gastric cancer, ASPN expression was not correlated with the prognosis $(p=0.104)$.

\section{Results}

\section{The Relationship between Clinicopathologic Features} and ASPN Expression

ASPN was expressed on either cancer cells or stromal cells in the gastric tumor environment (Fig. 1). ASPN was present mainly on stromal cells, especially on fibroblasts in tumor stroma, i.e., CAF. Some gastric tumors expressed ASPN on both cancer cells and stromal cells. Among the 296 cases of gastric cancer, 17 cases (5.8\%) showed ASPN-positive expression on cancer cells and 72 cases (24.3\%) showed ASPN-positive expression on stro- 


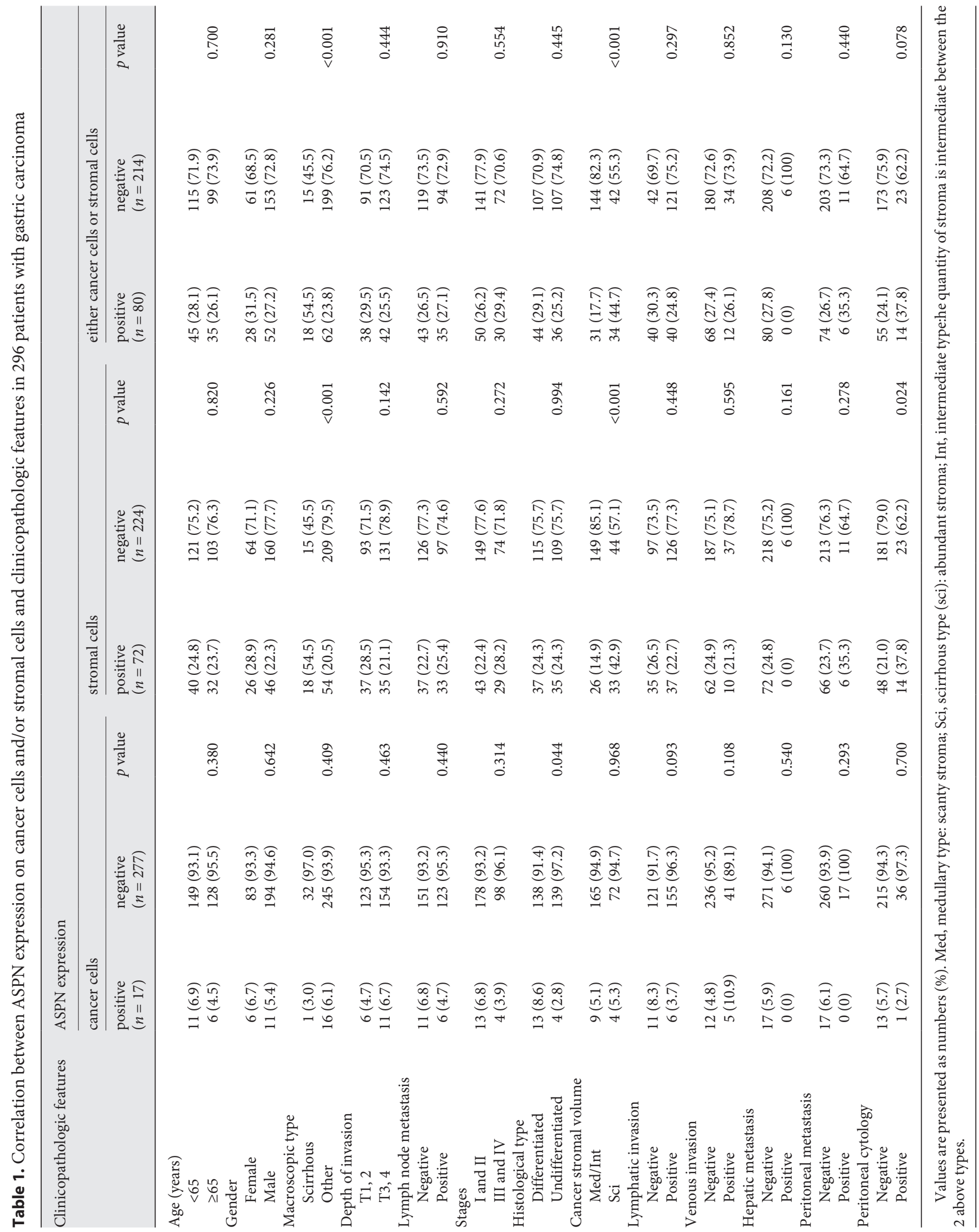


Table 2. Univariate analysis and multivariate analysis with respect to overall survival in 296 patients with gastric carcinoma

\begin{tabular}{|c|c|c|c|c|c|c|}
\hline \multirow[t]{2}{*}{ Parameter } & \multicolumn{3}{|c|}{ Univariate analysis } & \multicolumn{3}{|c|}{ Multivariate analysis } \\
\hline & risk ratio & $95 \% \mathrm{CI}$ & $p$ value & risk ratio & $95 \% \mathrm{CI}$ & $p$ value \\
\hline \multicolumn{7}{|c|}{ ASPN expression on cancer cells and/or stromal cells } \\
\hline Negative vs. positive & 1.056 & $0.665-1.676$ & 0.820 & & & \\
\hline \multicolumn{7}{|c|}{ ASPN expression on stromal cells } \\
\hline Negative vs. positive & 1.048 & $0.647-1.697$ & 0.849 & & & \\
\hline \multicolumn{7}{|c|}{ ASPN expression on cancer cells } \\
\hline Negative vs. positive & 1.098 & $0.479-2.515$ & 0.825 & & & \\
\hline \multicolumn{7}{|l|}{ Age (years) } \\
\hline$<65$ vs. $\geq 65$ & 1.713 & $1.114-2.635$ & 0.014 & 1.241 & $0.776-1.984$ & 0.367 \\
\hline \multicolumn{7}{|l|}{ Gender } \\
\hline Female vs. male & 1.096 & $0.696-1.728$ & 0.692 & & & \\
\hline \multicolumn{7}{|l|}{ Histological type } \\
\hline Intestinal diffuse & 1.832 & $1.184-2.834$ & 0.007 & 1.429 & $0.749-2.726$ & 0.279 \\
\hline \multicolumn{7}{|l|}{ Macroscopic type } \\
\hline Scirrhous vs. other & 9.091 & $5.663-14.594$ & $<0.001$ & 6.910 & $3.187-14.981$ & $<0.001$ \\
\hline \multicolumn{7}{|l|}{ Invasion depth (T stage) } \\
\hline I/II vs. III/IV & 5.323 & $3.312-8.555$ & $<0.001$ & 1.762 & $0.941-3.298$ & 0.077 \\
\hline \multicolumn{7}{|l|}{ Lymph node metastasis } \\
\hline Negative vs. positive & 6.884 & $4.096-11.568$ & $<0.001$ & 5.264 & $2.093-13.242$ & $<0.001$ \\
\hline \multicolumn{7}{|l|}{ Hepatic metastasis } \\
\hline Negative vs. positive & 8.131 & $3.518-18.793$ & $<0.001$ & 3.361 & $0.988-11.437$ & 0.052 \\
\hline \multicolumn{7}{|l|}{ Peritoneal dissemination } \\
\hline Negative vs. positive & 7.943 & $4.508-13.993$ & $<0.001$ & 3.138 & $1.469-6.706$ & 0.003 \\
\hline
\end{tabular}

mal cells. Eighty cases (27\%) showed ASPN-positive expression on either cancer cells or stromal cells, and 9 cases $(3 \%)$ showed the expression on both cancer cells and stromal cells.

The relationship between ASPN expression on cancer cells and/or stromal cells and clinicopathological features is described in Table 1. ASPN expression on stromal cells was significantly high in macroscopic scirrhous-type tumors $(p<0.001)$, histologically abundant stroma-type tumors $(p<0.001)$, and peritoneal cytology $(p=0.024)$. ASPN expression on cancer or stromal cells was also significantly high in macroscopic scirrhous-type tumors $(p<0.001)$ and histologically abundant stroma-type tumors $(p<0.001)$. In contrast, there was no significant correlation between ASPN expression on cancer cells and any of the clinicopathological features.

\section{Prognosis}

Figure 2a shows the Kaplan-Meier survival curve for all 296 patients; no significant difference in prognosis was observed between the ASPN-positive and ASPN-negative groups ( $p=0.819)$. Gastric cancer is histologically classified into 2 types by the Lauren classification as follows: diffuse type and intestinal type. Interestingly, in the 144 cases of diffuse-type gastric cancer, significantly poorer prognoses were observed in patients with ASPN-positive expression compared to those with ASPN-negative expression ( $p=0.043$; Fig. $2 b$ ), whereas in the 152 cases of intestinal-type gastric cancer the prognoses of patients with ASPN-positive expression were significantly better than those of patients with ASPN-negative expression ( $p=0.104$; Fig. $2 c)$.

The results of the univariate analysis with respect to overall survival for all 296 patients indicated that ASPN expression on cancer cells or stromal cells was not significantly associated with a poorer prognosis, whereas age $\geq 65$ years $(p=0.014)$, histologic diffuse type $(p=0.007)$, macroscopic scirrhous type $(p<0.001)$, invasion depth $(p<0.001)$, lymph node metastasis $(p<0.001)$, hepatic metastasis $(p<0.001)$, and peritoneal dissemination $(p<$ 0.001 ) were significantly associated with a poor prognosis. In the univariate analysis, macroscopic scirrhous type $(p<0.001)$, lymph node metastasis $(p<0.001)$, and peritoneal dissemination ( $p=0.003)$ were significantly associated with a poor prognosis (Table 2). Table 3 provides the results of the univariate analysis and multivariate analysis with respect to the overall survival of the 144 diffuse-type gastric cancer patients. In the univariate analy- 
Table 3. Univariate analysis and multivariate analysis respect to overall survival in 144 diffuse-type gastric cancer patients

\begin{tabular}{|c|c|c|c|c|c|c|}
\hline \multirow[t]{2}{*}{ Parameter } & \multicolumn{3}{|c|}{ Univariate analysis } & \multicolumn{3}{|c|}{ Multivariate analysis } \\
\hline & risk ratio & $95 \% \mathrm{CI}$ & $p$ value & risk ratio & $95 \% \mathrm{CI}$ & $p$ value \\
\hline \multicolumn{7}{|c|}{ ASPN expression on cancer cells and/or stromal cells } \\
\hline Negative vs. positive & 2.126 & $1.041-4.340$ & 0.038 & 1.686 & $1.020-2.786$ & 0.041 \\
\hline \multicolumn{7}{|c|}{ ASPN expression on stromal cells } \\
\hline Negative vs. positive & 1.811 & $1.011-3.246$ & 0.055 & & & \\
\hline \multicolumn{7}{|c|}{ ASPN expression on cancer cells } \\
\hline Negative vs. positive & 1.423 & $0.346-5.853$ & 0.625 & & & \\
\hline \multicolumn{7}{|l|}{ Age (years) } \\
\hline$<65$ vs. $\geq 65$ & 1.588 & $0.915-2.755$ & 0.100 & & & \\
\hline \multicolumn{7}{|l|}{ Gender } \\
\hline Female vs. male & 1.969 & $1.080-3.589$ & 0.027 & 0.991 & $0.617-1.592$ & 0.970 \\
\hline \multicolumn{7}{|l|}{ Macroscopic type } \\
\hline Scirrhous vs. other & 9.313 & $5.170-16.779$ & $<0.001$ & 4.609 & $2.602-8.164$ & $<0.001$ \\
\hline \multicolumn{7}{|l|}{ Invasion depth ( $\mathrm{T}$ stage) } \\
\hline I/II vs. III/IV & 7.862 & $3.689-16.753$ & $<0.001$ & 1.762 & $0.941-3.298$ & 0.077 \\
\hline \multicolumn{7}{|l|}{ Lymph node metastasis } \\
\hline Negative vs. positive & 11.128 & $4.733-26.166$ & $<0.001$ & 2.829 & $1.487-5.383$ & 0.002 \\
\hline \multicolumn{7}{|l|}{ Hepatic metastasis } \\
\hline Negative vs. positive & 5.632 & $1.749-18.140$ & $<0.001$ & 3.225 & $1.328-7.832$ & 0.010 \\
\hline \multicolumn{7}{|l|}{ Peritoneal dissemination } \\
\hline Negative vs. positive & 8.381 & $4.149-14.747$ & $<0.001$ & 3.164 & $1.328-7.832$ & $<0.001$ \\
\hline
\end{tabular}

sis, ASPN expression on cancer cells and/or stromal cells $(p=0.038)$, male gender $(p=0.027)$, macroscopic scirrhous type $(p<0.001)$, invasion depth $(p<0.001)$, lymph node metastasis $(p<0.001)$, hepatic metastasis $(p<0.001)$, and peritoneal dissemination $(p<0.001)$ were significantly associated with a poor prognosis. The results of the multivariate analysis showed that ASPN expression ( $p=$ $0.041)$, macroscopic scirrhous type $(p<0.001)$, lymph node metastasis $(p=0.002)$, hepatic metastasis $(p=0.010)$, and peritoneal dissemination $(p<0.001)$ were significantly correlated with overall survival in patients with diffuse-type gastric cancer.

\section{Discussion}

In the present study, ASPN was expressed on cancer cells and/or stromal cells in gastric tumors. It was unclear which types of cells expressed ASPN in tumor microenvironments. ASPN has been reported to be expressed mainly on stromal cells, especially CAF, in gastric cancer $[5,6,17]$, pancreatic cancer [7, 8], colorectal cancer [9], lung cancer [18], and prostate cancer [10, 16]. ASPN expression has also been detected on cancer cells of triplenegative breast cancer [11-13] and colorectal cancer [9]. Our present analyses demonstrated that ASPN was ex- pressed on cancer cells and/or stromal cells in 80 (27\%) of the 296 gastric cancers. Among these 80 cases, 72 cases and 17 cases were ASPN-positive on stromal cells and cancer cells, respectively, and 9 cases were ASPN-positive on both cells. These ASPN expression cases were significantly frequent in macroscopic scirrhous-type tumors, in abundant stroma tumors, and in peritoneal dissemination.

A characteristic of scirrhous-type gastric cancer is rapid proliferation with abundant stromal cells [19] and a high incidence of peritoneal dissemination [20, 21]. Because normal gastrointestinal tissues do not express ASPN [8], ASPN might be produced mainly by cancerspecific stromal cells such as activated fibroblasts, i.e., CAF. Those findings indicated that ASPN from CAF might be associated with the phenotype of scirrhous gastric cancer, one of the specific types of diffuse-type gastric cancer.

Gastric cancer is histologically classified into a diffuse type or an intestinal type by the Lauren classification [22]. In diffuse gastric cancer, cancer cells grow by poor adhesion, and a few cells infiltrate into the stroma, with the result that cancer cells are surrounded by stromal cells. Although our present analyses showed that prognoses were not significantly different between the ASPN-positive and ASPN-negative groups among the 296 patients, 
a significant difference in prognosis was revealed between these 2 groups among the 144 patients with diffuse-type gastric cancer. On the other hand, among those 144 cases of diffuse-type gastric cancer, a significantly poorer prognosis was observed in patients with ASPN-positive expression compared to those with ASPN-negative expression, but the prognoses of patients with ASPN-positive tumors were not significantly between these 2 groups among the 152 patients with intestinal-type gastric cancer.

It has been reported that ASPN promotes cancer cell proliferation and invasion by binding with CD44 or transforming growth factor (TGF)- $\beta /$ Smad $[4,9]$, which are frequently expressed in diffuse-type gastric cancer. Our prior investigation indicated that the gastric cancer cell line 44As3 promotes ASPN expression in CAF via a mechanism that functions through a CD44/Rac1-mediated axis [5]. ASPN has been reported to act as a negative regulator of TGF $\beta /$ Smad signaling, which shows suppressive effects against the proliferation of gastric cancer cells [23-27]. These findings suggest that ASPN might stimulate the progression of diffuse-type gastric cancer cells with CD44 or TGF $\beta 1 /$ Smad expression. ASPN might be a valuable therapeutic target in patients with diffuse-type gastric cancer.

Our multivariate analysis in patients with diffuse-type gastric cancer revealed that ASPN expression was an independent prognostic factor. ASPN from CAF may play a critical role in the progression of diffuse-type gastric cancer with CD44 or TGF $\beta 1 /$ Smad expression, resulting in a poor prognoses for patients with ASPN-positive expression.

We also identified 17 gastric cancer cases that had ASPN-positive expression on cancer cells. There was no significant correlation between ASPN expression on cancer cells and any of the clinicopathological features. ASPN was expressed on gastric cancer cells in relatively few cases, and it thus might not play an important role in the phenotype of gastric cancer.

One of limitations of this study is the histologic heterogeneity of gastric carcinoma. Gastric carcinoma frequently shows the histologic heterogeneity with coexistence of intestinal-type and diffuse-type adenocarcinomas, and the histologic type is determined based on the dominant element of histologic patterns as well as this study. The significance of ASPN in gastric cancer with histologic heterogeneity must be studied in the future. The other limitation of this study is the effect of postoperative chemotherapy. This study included GC patients in an advanced stage who underwent adjuvant or postop- erative chemotherapy. Since an analysis of the effect of chemotherapy would be important, we would like to examine the significance of postoperative chemotherapy in a future study.

In conclusion, ASPN was expressed mainly on stromal cells and partially on cancer cells in gastric cancer. ASPN expression on stromal cells and/or cancer cells might be a useful prognostic marker in patients with diffuse-type gastric cancer.

\section{Acknowledgment}

We thank Akiko Tsuda (Molecular Oncology and Therapeutics, Osaka City University Graduate School of Medicine) for technical support.

\section{Statement of Ethics}

The study protocol conformed to the ethical guidelines of the Declaration of Helsinki. This study was approved by the Osaka City University Ethics Committee (reference No. 924). Informed consent was obtained from all of the patients prior to entry.

\section{Conflict of Interest Statement}

There are no financial or other interests with regard to this paper that might be construed as a conflict of interests.

\section{Funding Sources}

This study was partially funded by KAKENHI (Grant-in-Aid for Scientific Research No. 18H02883 and 23390329) from the Ministry of Education, Science, Sports, Culture and Technology of Japan.

\section{Author Contributions}

M.Y. and T.H. designed and performed the experiments, cowrote this paper, and contributed equally. T.F., Y.Y., and N.K. were responsible for sample preparation. K.S., M.Y., A.S., S.K., S.N. and T.T. were responsible for data accumulation. G.T., N.K., and M.O. did the material sampling. M.O. reviewed this paper. All of the authors read and approved the final version of this work. 


\section{References}

1 Iozzo RV. The biology of the small leucinerich proteoglycans. Functional network of interactive proteins. J Biol Chem. 1999 Jul; 274(27):18843-6.

2 Henry SP, Takanosu M, Boyd TC, Mayne PM, Eberspaecher H, Zhou W, et al. Expression pattern and gene characterization of asporin. a newly discovered member of the leucinerich repeat protein family. J Biol Chem. 2001 Apr;276(15):12212-21.

3 Lorenzo P, Aspberg A, Onnerfjord P, Bayliss MT, Neame PJ, Heinegard D. Identification and characterization of asporin. a novel member of the leucine-rich repeat protein family closely related to decorin and biglycan. J Biol Chem. 2001 Apr;276(15):12201-11.

4 Zhan S, Li J, Ge W. Multifaceted Roles of Asporin in Cancer: current Understanding. Front Oncol. 2019 Sep;9:948.

5 Satoyoshi R, Kuriyama S, Aiba N, Yashiro M, Tanaka M. Asporin activates coordinated invasion of scirrhous gastric cancer and cancerassociated fibroblasts. Oncogene. 2015 Jan; 34(5):650-60.

6 Ding Q, Zhang M, Liu C. Asporin participates in gastric cancer cell growth and migration by influencing EGF receptor signaling. Oncol Rep. 2015 Apr;33(4):1783-90.

7 Wang L, Wu H, Wang L, Zhang H, Lu J, Liang $\mathrm{Z}$, et al. Asporin promotes pancreatic cancer cell invasion and migration by regulating the epithelial-to-mesenchymal transition (EMT) through both autocrine and paracrine mechanisms. Cancer Lett. 2017 Jul;398:24-36.

8 Turtoi A, Musmeci D, Wang Y, Dumont B, Somja J, Bevilacqua G, et al. Identification of novel accessible proteins bearing diagnostic and therapeutic potential in human pancreatic ductal adenocarcinoma. J Proteome Res. 2011 Sep;10(9):4302-13

9 Li H, Zhang Z, Chen L, Sun X, Zhao Y, Guo $\mathrm{Q}$, et al. Cytoplasmic Asporin promotes cell migration by regulating TGF- $\beta / \mathrm{Smad} 2 / 3$ pathway and indicates a poor prognosis in colorectal cancer. Cell Death Dis. 2019 Feb; 10(2):109.
10 Best CJ, Gillespie JW, Yi Y, Chandramouli GV, Perlmutter MA, Gathright Y, et al. Molecular alterations in primary prostate cancer after androgen ablation therapy. Clin Cancer Res. 2005 Oct;11(19 Pt 1):6823-34.

11 Castellana B, Escuin D, Peiró G, GarciaValdecasas B, Vázquez T, Pons C, et al. ASPN and GJB2 Are Implicated in the Mechanisms of Invasion of Ductal Breast Carcinomas. J Cancer. 2012;3:175-83.

12 Simkova D, Kharaishvili G, Korinkova G, Ozdian T, Suchánková-Kleplová T, Soukup T, et al. The dual role of asporin in breast cancer progression. Oncotarget. 2016 Aug;7(32): 52045-60.

13 Maris P, Blomme A, Palacios AP, Costanza B, Bellahcène A, Bianchi E, et al. Asporin Is a Fibroblast-Derived TGF- $\beta 1$ Inhibitor and a Tumor Suppressor Associated with Good Prognosis in Breast Cancer. PLoS Med. 2015 Sep;12(9):e1001871.

14 Japanese Gastric Cancer Association. Japanese gastric cancer treatment guidelines 2018 (5th edition). Gastric Cancer. 2021 Jan;24:121.

15 Japanese Gastric Cancer Association. Japanese classification of gastric carcinoma: 3rd English edition. Gastric Cancer. 2011 Jun; 14(2):101-12

16 Hurley PJ, Sundi D, Shinder B, Simons BW, Hughes RM, Miller RM, et al. Germline Variants in Asporin Vary by Race, Modulate the Tumor Microenvironment, and Are Differentially Associated with Metastatic Prostate Cancer. Clin Cancer Res. 2016 Jan;22(2):448-58.

17 Zhang Z, Li H, Zhao Y, Guo Q, Yu Y, Zhu S, et al. Asporin promotes cell proliferation via interacting with PSMD2 in gastric cancer. Front Biosci. 2019 Jun;24(6):1178-89.

18 Wang Y, Liu H, Bian Y, An J, Duan X, Wan J, et al. Low SCRIB expression in fibroblasts promotes invasion of lung cancer cells. Life Sci. 2020 Sep;256:117955.
19 Yashiro M, Hirakawa K. Cancer-stromal interactions in scirrhous gastric carcinoma. Cancer Microenviron. 2010 Jan;3(1):127-35.

20 Nakazawa K, Yashiro M, Hirakawa K. Keratinocyte growth factor produced by gastric fibroblasts specifically stimulates proliferation of cancer cells from scirrhous gastric carcinoma. Cancer Res. 2003 Dec;63(24):8848-52.

21 Tendo M, Yashiro M, Nakazawa K, Yamada N, Hirakawa K. Inhibitory effect of a selective cyclooxygenase inhibitor on the invasionstimulating activity of orthotopic fibroblasts for scirrhous gastric cancer cells. Cancer Sci. 2005 Jul;96(7):451-5.

22 Lauren P. The Two Histological Main Types of Gastric Carcinoma: Diffuse and So-Called Intestinal-Type Carcinoma. An Attempt at a Histo-Clinical Classification. Acta Pathol Microbiol Scand. 1965;64(1):31-49.

23 Nakajima M, Kizawa H, Saitoh M, Kou I, Miyazono K, Ikegawa S. Mechanisms for asporin function and regulation in articular cartilage. J Biol Chem. 2007 Nov;282(44):32185-92.

24 Kizawa H, Kou I, Iida A, Sudo A, Miyamoto Y, Fukuda A, et al. An aspartic acid repeat polymorphism in asporin inhibits chondrogenesis and increases susceptibility to osteoarthritis. Nat Genet. 2005 Feb;37(2):138-44.

25 Loughlin J. Polymorphism in signal transduction is a major route through which osteoarthritis susceptibility is acting. Curr Opin Rheumatol. 2005 Sep;17(5):629-33.

26 Engle SJ, Hoying JB, Boivin GP, Ormsby I, Gartside PS, Doetschman T. Transforming growth factor betal suppresses nonmetastatic colon cancer at an early stage of tumorigenesis. Cancer Res. 1999 Jul;59(14):3379-86

27 Miles FL, Tung NS, Aguiar AA, Kurtoglu S, Sikes RA. Increased TGF- $\beta 1$-mediated suppression of growth and motility in castrateresistant prostate cancer cells is consistent with Smad2/3 signaling. Prostate. 2012 Sep; 72(12):1339-50. 